\title{
Dual Jacobsthal Quaternions
}

\author{
Fügen Torunbalcı Aydın ${ }^{1 *}$
}

\begin{abstract}
In this paper, dual Jacobsthal quaternions were defined. Also, the relations between dual Jacobsthal quaternions which connected with Jacobsthal and Jacobsthal-Lucas numbers were investigated. Furthermore, Binet's formula, Honsberger identity, D'ocagne's identity, Cassini's identity and Catalan's identity for these quaternions were given.

Keywords: Jacobsthal number, Jacobsthal-Lucas number, Jacobsthal quaternion, dual Jacobsthal quaternion. 2010 AMS: 11R52, 20G20, 15A66, 11L10

${ }^{1}$ Yildiz Technical University, Faculty of Chemical and Metallurgical Engineering, Department of Mathematical Engineering, Davutpașa Campus, 34220, Esenler, Istanbul, Turkey

*Corresponding author:faydin@yildiz.edu.tr

Received: 27 January 2020, Accepted: 22 September 2020, Available online: 29 September 2020
\end{abstract}

\section{Introduction}

In 1843, Hamilton [1] introduced the set of quaternions which can be represented as

$$
H=\left\{q=q_{0}+i q_{1}+j q_{2}+k q_{3} \mid q_{0}, q_{1}, q_{2}, q_{3} \in \mathbb{R}\right\}
$$

where

$$
i^{2}=j^{2}=k^{2}=-1, \quad i j=-j i=k, \quad j k=-k j=i, \quad k i=-i k=j .
$$

After the work of Hamilton, several authors worked on different quaternions and their generalizations. ([2]-[22]).

In 1973, Sloane [23] introduced the set of Jacobsthal numbers.

Further, in 1988, Horadam [24]-[25] defined the Jacobsthal and Jacobsthal-Lucas sequences $\left\{J_{n}\right\}$ and $\left\{j_{n}\right\}$ with the recurrence relations respectively, as follows

$$
J_{0}=0, \quad J_{1}=1, \quad J_{n}=J_{n-1}+2 J_{n-2}, \text { for } n \geq 2
$$

and

$$
j_{0}=2, \quad j_{1}=1, \quad j_{n}=j_{n-1}+2 j_{n-2}, \text { for } n \geq 2,
$$

In 1996, Horadam studied on the Jacobsthal and Jacobsthal-Lucas sequences and he gave Cassini-like formula as follows [26]

$$
J_{n+1} J_{n-1}-J_{n}^{2}=(-1)^{n} \cdot 2^{n-1}
$$




$$
j_{n+1} j_{n-1}-j_{n}^{2}=3^{2} \cdot(-1)^{n+1} \cdot 2^{n-1}
$$

The first eleven terms of Jacobsthal sequence $\left\{J_{n}\right\}$ are $0,1,1,3,5,11,21,43,85,171$ and 341 . This sequence is given by the formula

$$
J_{n}=\frac{2^{n}-(-1)^{n}}{3}
$$

The first eleven terms of Jacobsthal-Lucas sequence $\left\{j_{n}\right\}$ are $2,1,5,7,17,31$

$65,127,257,511$ and 1025 . This sequence is given by the formula

$$
j_{n}=2^{n}+(-1)^{n}
$$

Also, we can see the matrix representations of Jacobsthal and Jacobsthal-Lucas numbers in [27],[28]. The members of these integer sequences can also be obtained in different ways: Binet formulae or matrix method by Köken and Bozkurt [27]-[28]. Several authors worked on Jacobsthal numbers and polynomials in [29]-[32].

In 2015, Szynal-Liana and Włoch [33] defined the Jacobsthal quaternions and the Jacobsthal- Lucas quaternions respectively as follows

$$
J Q_{n}=J_{n}+i J_{n+1}+j J_{n+2}+k J_{n+3},
$$

and

$$
J L Q_{n}=j_{n}+i j_{n+1}+j j_{n+2}+k j_{n+3} .
$$

where

$$
i^{2}=j^{2}=k^{2}=-1, i j=-j i=k, \quad j k=-k j=i, \quad k i=-i k=j .
$$

In 2017, Torunbalcı Aydın and Yüce [34] given a new approach to Jacobsthal quaternions. Furthermore, some relations between Jacobsthal and Jacobsthal-Lucas quaternions are given in [34].

In 2017, Taşç1 [35] defined k-Jacobsthal and k-Jacobsthal-Lucas quaternions as follows

$$
Q J_{k, n}=J_{k, n}+i_{1} J_{k, n+1}+i_{2} J_{k, n+2}+i_{3} J_{k, n+3}
$$

and

$$
Q j_{k, n}=j_{k, n}+i_{1} j_{k, n+1}+i_{2} j_{k, n+2}+i_{3} j_{k, n+3}
$$

where

$$
i_{1}^{2}=i_{2}^{2}=i_{3}^{2}=i_{1} i_{2} i_{3}=-1
$$

In 2017, Cerda-Morales [36] worked on identities of third order Jacobsthal quaternions.

In 2018, Cerda-Morales [37] defined fourth-order Jacobsthal and Jacobsthal-Lucas quaternions as follows

$$
Q J_{n}{ }^{(4)}=J_{n}{ }^{(4)}+i J_{n+1}{ }^{(4)}+j J_{n+2}{ }^{(4)}+k J_{n+3}{ }^{(4)}
$$

and

$$
Q j_{n}{ }^{(4)}=j_{n}{ }^{(4)}+i j_{n+1}{ }^{(4)}+j j_{n+2}{ }^{(4)}+k j_{n+3}{ }^{(4)}
$$

In this paper, dual Jacobsthal and dual Jacobsthal-Lucas quaternions will be defined as follows

$$
J_{D}=\left\{D_{n}^{J}=J_{n}+i J_{n+1}+j J_{n+2}+k J_{n+3} \mid J_{n}, n-t h \text { Jacobsthal number }\right\}
$$


and

$$
j_{D}=\left\{D_{n}^{j}=j_{n}+i j_{n+1}+j j_{n+2}+k j_{n+3} \mid j_{n}, n-t h \text { Jacobsthal-Lucas number }\right\}
$$

where

$$
i^{2}=j^{2}=k^{2}=i j k=0, i j=-j i=j k=-k j=k i=-i k=0 .
$$

All the studies on Jacobsthal quaternions are summarized in Table 1.

Table 1. Types of Jacobsthal quaternions [33]-[35].

\begin{tabular}{ccc}
\hline & Definition & Multiplication Rules \\
\hline $\begin{array}{c}\text { Jacobsthal } \\
\text { quaternion }\end{array}$ & $\begin{array}{c}J Q_{n}=\left(J_{n}, J_{n+1}, J_{n+2}, J_{n+3}\right) \\
J_{n}=J_{n-1}+2 J_{n-2}, J_{1}=J_{2}=1\end{array}$ & $\begin{array}{c}(1, i, j, k), i^{2}=j^{2}=k^{2}=-1 \\
i j=-j i=k, j k=-k j=i \\
k i=-i k=j\end{array}$ \\
\hline $\begin{array}{c}\text { k-Jacobsthal } \\
\text { quaternion }\end{array}$ & $\begin{array}{c}Q J_{k, n}=\left(J_{k, n}, J_{k, n+1}, J_{k, n+2}, J_{k, n+3}\right) \\
Q J_{k, n+2}=k Q J_{k, n+1}+2 Q J_{k, n}\end{array}$ & $i_{1}^{2}=i_{2}^{2}=i_{3}^{2}=i_{1} i_{2} i_{3}=-1$ \\
\hline $\begin{array}{c}\text { Dual Jacobsthal } \\
\text { quaternion }\end{array}$ & $D_{n}=\left(J_{n}, J_{n+1}, J_{n+2}, J_{n+3}\right)$ & $\begin{array}{c}(1, i, j, k) i^{2}=j^{2}=k^{2}=i j k=0 \\
i j=-j i=j k=-k j=k i=-i k=0\end{array}$
\end{tabular}

\section{Dual Jacobsthal Quaternions}

In this section, the dual Jacobsthal quaternions will be defined. Also, the relations between dual Jacobsthal quaternions which connected with Jacobsthal and Jacobsthal-Lucas numbers were investigated.

Dual Jacobsthal quaternions is defined by relation recurrence (1.2) as follows

$$
J_{D}=\left\{D_{n}^{J}=J_{n}+i J_{n+1}+j J_{n+2}+k J_{n+3} \mid J_{n}, n-t h \text { Jacobsthal number }\right\}
$$

where

$$
i^{2}=j^{2}=k^{2}=i j k=0, i j=-j i=j k=-k j=k i=-i k=0 .
$$

Also, the dual Jacobsthal-Lucas quaternion is defined by relation recurrence (1.3) as follows

$$
\begin{aligned}
& j_{D}=\left\{D_{n}^{j}=j_{n}+i j_{n+1}+j j_{n+2}+k j_{n+3} \mid j_{n}, n-t h \text { Jacobsthal-Lucas number }\right\}, \\
& i^{2}=j^{2}=k^{2}=i j k=0, i j=-j i=j k=-k j=k i=-i k=0 .
\end{aligned}
$$

Let $D_{n}^{J_{1}}$ and $D_{n}^{J_{2}}$ be n-th terms of the dual Jacobsthal quaternion sequence $\left(D_{n}^{J_{1}}\right)$ and $\left(D_{n}^{J_{2}}\right)$ such that

$$
D_{n}^{J_{1}}=J_{n}+i J_{n+1}+j J_{n+2}+k J_{n+3}
$$

and

$$
D_{n}^{J_{2}}=K_{n}+i K_{n+1}+j K_{n+2}+k K_{n+3}
$$


Then, the addition and subtraction of the dual Jacobsthal quaternions is defined by

$$
\begin{aligned}
D_{n}^{J_{1}} \pm D_{n}^{J_{2}}= & \left(J_{n}+i J_{n+1}+j J_{n+2}+k J_{n+3}\right) \\
& \pm\left(K_{n}+i K_{n+1}+j K_{n+2}+k K_{n+3}\right) \\
= & \left(J_{n} \pm K_{n}\right)+i\left(J_{n+1} \pm K_{n+1}\right)+j\left(J_{n+2} \pm K_{n+2}\right) \\
& +k\left(J_{n+3} \pm K_{n+3}\right) .
\end{aligned}
$$

Multiplication of the dual Jacobsthal quaternions is defined by

$$
\begin{aligned}
D_{n}^{J_{1}} D_{n}^{J_{2}}= & \left(J_{n}+i J_{n+1}+j J_{n+2}+k J_{n+3}\right) \\
& \left(K_{n}+i K_{n+1}+j K_{n+2}+k K_{n+3}\right) \\
= & \left(J_{n} K_{n}\right)+i\left(J_{n} K_{n+1}+J_{n+1} K_{n}\right)+j\left(J_{n} K_{n+2}+J_{n+2} K_{n}\right) \\
& +k\left(J_{n} K_{n+3}+J_{n+3} K_{n}\right) .
\end{aligned}
$$

The scalar and the vector part of $D_{n}^{J}$ which is the n-th term of the dual Jacobsthal quaternion $\left(D_{n}^{J}\right)$ are denoted by

$$
S_{D_{n}^{J}}=J_{n} \text { and } V_{D_{n}^{J}}=i J_{n+1}+j J_{n+2}+k J_{n+3} .
$$

Thus, the dual Jacobsthal quaternion $D_{n}^{J}$ is given by $D_{n}^{J}=S_{D_{n}^{J}}+V_{D_{n}^{J}}$.

Then, relation (2.7) is defined by

$$
D_{n}^{J_{1}} D_{n}^{J_{2}}=S_{D_{n}^{J_{1}}} \cdot S_{D_{n}^{J_{2}}}+S_{D_{n}^{J_{1}}} \cdot V_{D_{n}^{J_{2}}}+S_{D_{n}^{J_{2}}} \cdot V_{D_{n}^{J_{1}}}
$$

The conjugate of the dual Jacobsthal quaternion $D_{n}^{J}$ is denoted by $\overline{D_{n}^{J}}$ and it is

$$
\overline{D_{n}^{J}}=J_{n}-i J_{n+1}-j J_{n+2}-k J_{n+3} .
$$

The norm of $D_{n}^{J}$ is defined as

$$
N_{D_{n}^{J}}=\left\|D_{n}^{J}\right\|^{2}=D_{n}^{J} \overline{D_{n}^{J}}=J_{n}^{2} .
$$

Then, we give the following theorem using statements $(2.1),(2.2)$ and

$$
\left\{\begin{array}{l}
J_{n} J_{n+1}+2 J_{n-1} J_{n}=J_{2 n}, \\
J_{n} J_{m+1}+2 J_{n-1} J_{m}=J_{n+m}, \\
J_{n+1}+2 J_{n-1}=j_{n}, \\
J_{n} j_{n}=J_{2 n} .
\end{array}\right.
$$

Theorem 2.1. Let $J_{n}$ and $D_{n}^{J}$ be the $n$-th terms of the Jacobsthal sequence $\left(J_{n}\right)$ and the dual Jacobsthal quaternion sequence $\left(D_{n}^{J}\right)$, respectively. In this case, for $n \geq 1$ we can give the following relations:

$$
D_{n}^{J}+\overline{D_{n}^{J}}=2 J_{n},
$$

$$
\left(D_{n}^{J}\right)^{2}+D_{n}^{J} \overline{D_{n}^{J}}=2 J_{n} D_{n}^{J},
$$

$$
D_{n+1}^{J}+2 D_{n}^{J}=D_{n+2}^{J},
$$

$$
D_{n}^{J}-i D_{n+1}^{J}-j D_{n+2}^{J}-k D_{n+3}^{J}=J_{n},
$$

Proof. Proof of four equality can easily be done by the equations

$$
D_{n}^{J}=J_{n}+i J_{n+1}+j J_{n+2}+k J_{n+3},
$$




$$
D_{n+1}^{J}=J_{n+1}+i J_{n+2}+j J_{n+3}+k J_{n+4}
$$

(2.13):

$$
\begin{aligned}
D_{n}^{J}+\overline{D_{n}^{J}=} & \left(J_{n}+i J_{n+1}+j J_{n+2}+k J_{n+3}\right) \\
& +\left(J_{n}-i J_{n+1}-j J_{n+2}-k J_{n+3}\right) \\
= & \left(J_{n}+J_{n}\right)+i\left(J_{n+1}-J_{n+1}\right)+j\left(J_{n+2}-J_{n+2}\right) \\
& +k\left(J_{n+3}-J_{n+3}\right) \\
= & 2 J_{n} .
\end{aligned}
$$

(2.14):

$$
\begin{aligned}
\left(D_{n}^{J}\right)^{2}+D_{n}^{J} \overline{D_{n}^{J}=} & \left(J_{n}+i J_{n+1}+j J_{n+2}+k J_{n+3}\right) \\
& \left(J_{n}+i J_{n+1}+j J_{n+2}+k J_{n+3}\right) \\
& +\left(J_{n}+i J_{n+1}+j J_{n+2}+k J_{n+3}\right) \\
& \left(J_{n}-i J_{n+1}-j J_{n+2}-k J_{n+3}\right) \\
= & \left(J_{n} J_{n}\right)+i\left(J_{n} J_{n+1}+J_{n+1} J_{n}\right)+j\left(J_{n} J_{n+2}+J_{n+2} J_{n}\right) \\
& +k\left(J_{n} J_{n+3}+J_{n+3} J_{n}\right) \\
& +J_{n} J_{n}+i\left(-J_{n} J_{n+1}+J_{n+1} J_{n}\right) \\
& +j\left(-J_{n} J_{n+2}+J_{n+2} J_{n}\right) \\
& +k\left(-J_{n} J_{n+3}+J_{n+3} J_{n}\right) \\
= & 2 J_{n} J_{n}+2 i J_{n} J_{n+1}+2 j J_{n} J_{n+2}+2 k J_{n} J_{n+3} \\
= & 2 J_{n}\left(J_{n}+i J_{n+1}+j J_{n+2}+k J_{n+3}\right) \\
= & 2 J_{n} D_{n}^{J}
\end{aligned}
$$

(2.15):

$$
\begin{aligned}
D_{n+1}^{J}+2 D_{n}^{J}= & \left(J_{n+1}+i J_{n+2}+j J_{n+3}+k J_{n+4}\right) \\
& +2\left(J_{n}+i J_{n+1}+j J_{n+2}+k J_{n+3}\right) \\
= & \left(J_{n+1}+2 J_{n}\right)+i\left(J_{n+2}+2 J_{n+1}\right)+j\left(J_{n+3}+2 J_{n+2}\right) \\
& +k\left(J_{n+4}+2 J_{n+3}\right) \\
= & J_{n+2}+i J_{n+3}+j J_{n+4}+k J_{n+5} \\
= & D_{n+2}^{J} .
\end{aligned}
$$

(2.16):

$$
\begin{aligned}
D_{n}^{J}-i D_{n+1}^{J}-j D_{n+2}^{J}-k D_{n+3}^{J} & =\left(J_{n}+i J_{n+1}+j J_{n+2}+k J_{n+3}\right) \\
& -i\left(J_{n+1}+i J_{n+2}+j J_{n+3}+k J_{n+4}\right) \\
& -j\left(J_{n+2}+i J_{n+3}+j J_{n+4}+k J_{n+5}\right) \\
& -k\left(J_{n+3}+i J_{n+4}+j J_{n+5}+k J_{n+6}\right) \\
& =J_{n} .
\end{aligned}
$$

Theorem 2.2. Let $D_{n}^{J}$ and $D_{n}^{j}$ be the $n$-th terms of the dual Jacobsthal quaternion sequence $\left(D_{n}^{J}\right)$ and the dual Jacobsthal-Lucas quaternion sequence $\left(D_{n}^{j}\right)$, respectively. The following relations are satisfied

$$
\begin{aligned}
& D_{n+1}^{J}+2 D_{n-1}^{J}=D_{n}^{j}, \\
& 2 D_{n+1}^{J}-D_{n}^{J}=D_{n}^{j} .
\end{aligned}
$$

Proof. (2.19): From equations (2.17), (2.18) and identity between Jacobsthal number and Jacobsthal-Lucas number $j_{n}=J_{n+1}+2 J_{n-1}$, it follows that

$$
\begin{aligned}
D_{n+1}^{J}+2 D_{n-1}^{J}= & \left(J_{n+1}+i J_{n+2}+j J_{n+3}+k J_{n+4}\right) \\
& +2\left(J_{n-1}+i J_{n}+j J_{n+1}+k J_{n+2}\right) \\
= & \left(J_{n+1}+2 J_{n-1}\right)+i\left(J_{n+2}+2 J_{n}\right) \\
& +j\left(J_{n+3}+2 J_{n+1}\right)+k\left(J_{n+4}+2 J_{n+2}\right) \\
= & j_{n}+i j_{n+1}+j j_{n+2}+k j_{n+3} \\
= & D_{n}^{j} .
\end{aligned}
$$


(2.20): Using the identity between Jacobsthal number and Jacobsthal -Lucas number $J_{n}+j_{n}=2 J_{n+1}$, we get

$$
\begin{aligned}
2 D_{n+1}^{J}-D_{n}^{J}= & 2\left(J_{n+1}+i J_{n+2}+j J_{n+3}+k J_{n+4}\right) \\
& -\left(J_{n}+i J_{n+1}+j J_{n+2}+k J_{n+3}\right) \\
= & \left(2 J_{n+1}-J_{n}\right)+i\left(J_{n+2}-J_{n+1}\right) \\
& +j\left(2 J_{n+3}-J_{n+2}\right)+k\left(2 J_{n+4}-J_{n+3}\right) \\
= & j_{n}+i j_{n+1}+j j_{n+2}+k j_{n+3} \\
= & D_{n}^{j} .
\end{aligned}
$$

Theorem 2.3. Let $D_{n}^{J}$ be the $n$-th term of the dual Jacobsthal quaternion sequence $\left(D_{n}^{J}\right)$ and $\overline{D_{n}^{J}}$ be conjugate of $D_{n}^{J}$. Then, we can give the following relations between these quaternions:

$$
\begin{aligned}
& \left(D_{n}^{J}\right)^{2}=2 J_{n} D_{n}^{J}-J_{n}^{2}, \\
& \left(D_{n}^{J}\right)^{2}+2\left(D_{n-1}^{J}\right)^{2}=2 D_{2 n-1}^{J}-J_{2 n-1}, \\
& D_{n}^{J} \overline{D_{n}^{J}}+2 D_{n-1}^{J} \overline{D_{n-1}^{J}}=J_{n}^{2}+2 J_{n-1}^{2}=J_{2 n-1}, \\
& D_{n+1}^{J} \overline{D_{n+1}^{J}}+2 D_{n}^{J} \overline{D_{n}^{J}}=J_{n+1}^{2}+2 J_{n}^{2}=J_{2 n+1} \text {, } \\
& D_{n+1}^{J} \overline{D_{n+1}^{J}}-2 D_{n}^{J} \overline{D_{n}^{J}}=J_{n+1}^{2}-2 J_{n}^{2}=J_{2 n+1}-4 J_{n}^{2}
\end{aligned}
$$

Proof. It can be proved easily by using (2.10). Now, we will prove first two equalities

$$
\begin{aligned}
\left(D_{n}^{J}\right)^{2}= & J_{n} J_{n}+i\left(J_{n} J_{n+1}+J_{n+1} J_{n}\right)+j\left(J_{n} J_{n+2}+J_{n+2} J_{n}\right) \\
& +k\left(J_{n} J_{n+3}+J_{n+3} J_{n}\right) \\
= & 2 J_{n}\left(J_{n}+i J_{n+1}+j J_{n+2}+k J_{n+3}\right)-J_{n} J_{n} \\
= & 2 J_{n} D_{n}^{J}-J_{n}^{2} . \\
\left(D_{n}^{J}\right)^{2}+2\left(D_{n-1}^{J}\right)^{2}= & J_{n}^{2}+2 i\left(J_{n} J_{n+1}\right)+2 j\left(J_{n} J_{n+2}\right)+2 k\left(J_{n} J_{n+3}\right) \\
& +2\left(J_{n-1}^{2}\right)+4 i\left(J_{n-1} J_{n}\right)+4 j\left(J_{n-1} J_{n+1}\right) \\
& +4 k\left(J_{n-1} J_{n+2}\right) \\
= & \left(J_{n}^{2}+2 J_{n-1}^{2}\right)+i\left(2 J_{n} J_{n+1}+4 J_{n-1} J_{n}\right) \\
& +j\left(2 J_{n} J_{n+2}+4 J_{n-1} J_{n+1}\right) \\
& +k\left(2 J_{n} J_{n+3}+4 J_{n-1} J_{n+2}\right) \\
= & J_{2 n-1}+2 i J_{2 n}+2 j J_{2 n+1}+2 k J_{2 n+2} \\
= & 2 D_{2 n-1}^{J}-J_{2 n-1} .
\end{aligned}
$$

We can prove last three equalities by using equation (2.12) as follows:

$$
\begin{aligned}
& D_{n}^{J} \overline{D_{n}^{J}}+2 D_{n-1}^{J} \cdot \overline{D_{n-1}^{J}}=J_{n}^{2}+2 J_{n-1}^{2}=J_{2 n-1}, \\
& D_{n+1}^{J} \overline{D_{n+1}^{J}}+2 D_{n}^{J} \overline{D_{n}^{J}}=J_{n+1}^{2}+2 J_{n}^{2}=J_{2 n+1}, \\
& D_{n+1}^{J} \overline{D_{n+1}^{J}}-2 D_{n}^{J} \overline{D_{n}^{J}}=J_{n+1}^{2}-2 J_{n}^{2}=J_{2 n+1}-4 J_{n}^{2}
\end{aligned}
$$

where identities $J_{m} J_{n+1}+2 J_{m-1} J_{n}=J_{m+n}$ and $J_{n}^{2}+2 J_{n-1}^{2}=J_{2 n-1}$ were used.

Theorem 2.4. Let $D_{n}^{J}$ be the $n$-th term of dual Jacobsthal quaternion sequence $\left(D_{n}^{J}\right)$. Then, we have the following identities

$$
\begin{aligned}
& \sum_{s=1}^{n} D_{s}^{J}=\frac{1}{2}\left[D_{n+2}^{J}-D_{2}^{J}\right], \\
& \sum_{s=0}^{p} D_{n+s}^{J}=\frac{1}{2}\left[D_{n+p+2}^{J}-D_{n+1}^{J}\right], \\
& \sum_{s=1}^{n} D_{2 s-1}^{J}=\frac{2 D_{2 n}^{J}}{3}+\frac{1}{3}\left[n\left(2 D_{2}^{J}-D_{3}^{J}\right)-2 D_{0}^{J}\right],
\end{aligned}
$$




$$
\sum_{s=1}^{n} D_{2 s}^{J}=\frac{2 D_{2 n+1}^{J}}{3}-\frac{1}{3}\left[n\left(2 D_{2}^{J}-D_{3}^{J}\right)+2 D_{1}^{J}\right]
$$

Proof. (2.22) Hence, we can write

$$
\begin{aligned}
\sum_{s=1}^{n} D_{s}^{J} & =\sum_{s=1}^{n} J_{s}+i \sum_{s=1}^{n} J_{s+1}+j \sum_{s=1}^{n} J_{s+2}+k \sum_{s=1}^{n} J_{s+3} \\
& =\frac{1}{2}\left[\left(J_{n+2}-1\right)+i\left(J_{n+3}-3\right)+j\left(J_{n+4}-5\right)+k\left(J_{n+5}-11\right)\right] \\
& =\frac{1}{2}\left[\left(J_{n+2}-J_{2}\right)+i\left(J_{n+3}-J_{3}\right)+j\left(J_{n+4}-J_{4}\right)+k\left(J_{n+5}-J_{5}\right)\right] \\
& =\frac{1}{2}\left[J_{n+2}+i J_{n+3}+j J_{n+4}+k J_{n+5}-\left(J_{2}+i J_{3}+j J_{4}+k J_{5}\right)\right] \\
& =\frac{1}{2}\left[D_{n+2}^{J}-D_{2}^{J}\right] .
\end{aligned}
$$

(2.23) Hence, we can write

$$
\begin{aligned}
\sum_{s=0}^{p} D_{n+s}^{J}= & \sum_{s=0}^{p} J_{n+s}+i \sum_{s=0}^{p} J_{n+s+1}+j \sum_{s=0}^{p} J_{n+s+2}+k \sum_{s=0}^{p} J_{n+s+3} \\
= & \frac{1}{2}\left[\left(J_{n+p+2}-J_{n+1}\right)+i\left(J_{n+p+3}-J_{n+2}\right)+j\left(J_{n+p+4}-J_{n+3}\right)\right] \\
& +\frac{1}{2}\left[k\left(J_{n+p+5}-J_{n+4}\right)\right] \\
= & \frac{1}{2}\left[J_{n+p+2}+i J_{n+p+3}+j J_{n+p+4}+k J_{n+p+5}\right. \\
& \left.-\left(J_{n+1}+i J_{n+2}+j J_{n+3}+k J_{n+4}\right)\right] \\
= & \frac{1}{2}\left[D_{n+p+2}^{J}-D_{n+1}^{J}\right] .
\end{aligned}
$$

(2.24): Using $\sum_{i=0}^{n-1} J_{2 i+1}=\frac{2 J_{2 n}+n}{3}$ and $\sum_{i=0}^{n} J_{2 i}=\frac{2 J_{2 n+1}-n-2}{3}$, we get

$$
\begin{aligned}
\sum_{s=1}^{n} D_{2 s-1}^{J}= & \left(J_{1}+J_{3}+\ldots+J_{2 n-1}\right)+i\left(J_{2}+J_{4}+\ldots+J_{2 n}\right) \\
& +j\left(J_{3}+J_{5}+\ldots+J_{2 n+1}\right)+k\left(J_{4}+J_{6}+\ldots+J_{2 n+2}\right) \\
= & \frac{\left(2 J_{2 n}+n\right)}{3}+i \frac{\left(2 J_{2 n+1}-n-2\right)}{3}+j \frac{\left(2 J_{2 n+2}+n-2\right)}{3} \\
& +k \frac{\left(2 J_{2 n+3}-n-6\right)}{3} \\
= & \frac{2}{3}\left[J_{2 n}+i J_{2 n+1}+j J_{2 n+2}+k J_{2 n+3}\right] \\
& +\frac{1}{3}[n(1-i+j-k)-2(i+j+3 k)] \\
= & \frac{2 D_{2 n}^{J}}{3}+\frac{1}{3}\left[n\left(2 D_{2}^{J}-D_{3}^{J}\right)-2 D_{0}^{J}\right] .
\end{aligned}
$$


(2.25): Using $\sum_{i=0}^{n} J_{2 i}=\frac{2 J_{2 n+1}-n-2}{3}$ we obtain

$$
\begin{aligned}
\sum_{s=1}^{n} D_{2 s}^{J}= & \left(J_{2}+J_{4}+\ldots+J_{2 n}\right)+i\left(J_{3}+J_{5}+\ldots+J_{2 n+1}\right) \\
& +j\left(J_{4}+J_{6}+\ldots+J_{2 n+2}\right)+k\left(J_{5}+J_{7}+\ldots+J_{2 n+3}\right) \\
= & \frac{\left(2 J_{2 n+1}-n-2\right)}{3}+i \frac{\left(2 J_{2 n+2}+n-2\right)}{3}+j \frac{\left(2 J_{2 n+3}-n-6\right)}{3} \\
& +k \frac{\left(2 J_{2 n+4}+n-10\right)}{3} \\
= & \frac{2}{3}\left[J_{2 n+1}+i J_{2 n+2}+j J_{2 n+3}+k J_{2 n+4}\right] \\
& +\frac{1}{3}[-n(1-i+j-k)-2(1+i+3 j+5 k)] \\
= & \frac{2 D_{2 n+1}^{J}}{3}-\frac{1}{3}\left[n\left(2 D_{2}^{J}-D_{3}^{J}\right)+2 D_{1}^{J}\right] .
\end{aligned}
$$

Theorem 2.5. Let $D_{n}^{J}$ and $D_{n}^{j}$ be the $n$-th terms of the dual Jacobsthal quaternion sequence $\left(D_{n}^{J}\right)$ and the dual Jacobsthal-Lucas quaternion sequence $\left(D_{n}^{j}\right)$, respectively. Then, we have

$$
\begin{aligned}
& D_{n}^{j} \overline{D_{n}^{J}}-\overline{D_{n}^{j}} D_{n}^{J}=2\left[J_{n} D_{n}^{j}-j_{n} D_{n}^{J}\right], \\
& D_{n}^{j} \overline{D_{n}^{J}}+\overline{D_{n}^{j}} D_{n}^{J}=2 j_{n} J_{n}=2 J_{2 n}, \\
& D_{n}^{j} D_{n}^{J}-\overline{D_{n}^{j}} \overline{D_{n}^{J}}=2\left[D_{n}^{j} J_{n}+D_{n}^{J} j_{n}-2 J_{2 n}\right], \\
& D_{n}^{j} D_{n}^{J}+\overline{D_{n}^{j}} \overline{D_{n}^{J}}=2 J_{2 n} .
\end{aligned}
$$

Proof. (2.26):

$$
\begin{aligned}
D_{n}^{j} \overline{D_{n}^{J}}-\overline{D_{n}^{j}} D_{n}^{J}= & \left(j_{n}+i j_{n+1}+j j_{n+2}+k j_{n+3}\right) \\
& \left(J_{n}-i J_{n+1}-j J_{n+2}-k J_{n+3}\right) \\
& -\left(j_{n}-i j_{n+1}-j j_{n+2}-k j_{n+3}\right) \\
& \left(J_{n}+i J_{n+1}+j J_{n+2}+k J_{n+3}\right) \\
= & \left(j_{n} J_{n}-j_{n} J_{n}\right) \\
& +2 i\left(j_{n+1} J_{n}-j_{n} J_{n+1}\right) \\
& +2 j\left(j_{n+2} J_{n}-j_{n} J_{n+2}\right) \\
& +2 k\left(j_{n+3} J_{n}-j_{n} J_{n+3}\right) \\
= & 2 J_{n}\left(j_{n}+i j_{n+1}+j j_{n+2}+k j_{n+3}\right) \\
& -2 j_{n}\left(J_{n}+i J_{n+1}+j J_{n+2}+k J_{n+3}\right) \\
= & 2\left[J_{n} D_{n}^{j}-j_{n} D_{n}^{J}\right] .
\end{aligned}
$$

(2.27):

$$
\begin{aligned}
D_{n}^{j} \overline{D_{n}^{J}}+\overline{D_{n}^{j}} D_{n}^{J}= & \left(j_{n}+i j_{n+1}+j j_{n+2}+k j_{n+3}\right) \\
& \left(J_{n}-i J_{n+1}-j J_{n+2}-k J_{n+3}\right) \\
& +\left(j_{n}-i j_{n+1}-j j_{n+2}-k j_{n+3}\right) \\
& \left(J_{n}+i J_{n+1}+j J_{n+2}+k J_{n+3}\right) \\
= & j_{n}\left[J_{n}-i J_{n+1}-j J_{n+2}-k J_{n+3}\right] \\
& +\left(i j_{n+1}+j j_{n+2}+k j_{n+3}\right) J_{n} \\
& +j_{n}\left[J_{n}+i J_{n+1}+j J_{n+2}+k J_{n+3}\right] \\
& +\left(-i j_{n+1}-j j_{n+2}-k j_{n+3}\right) J_{n} \\
= & 2 j_{n} J_{n}=2 J_{2 n} .
\end{aligned}
$$


(2.28):

$$
\begin{aligned}
D_{n}^{j} D_{n}^{J}-\overline{D_{n}^{j}} \overline{D_{n}^{J}=} & \left(j_{n}+i j_{n+1}+j j_{n+2}+k j_{n+3}\right) \\
& \left(J_{n}+i J_{n+1}+j J_{n+2}+k J_{n+3}\right) \\
& -\left(j_{n}-i j_{n+1}-j j_{n+2}-k j_{n+3}\right) \\
& \left(J_{n}-i J_{n+1}-j J_{n+2}-k J_{n+3}\right) \\
= & \left(j_{n} J_{n}-j_{n} J_{n}\right)+2\left(i j_{n+1}+j j_{n+2}+k j_{n+3}\right) J_{n} \\
& +2\left(j_{n}+i j_{n+1}+j j_{n+2}+k j_{n+3}\right) J_{n}-2 j_{n} J_{n} \\
= & 2\left(D_{n}^{j} J_{n}+D_{n}^{J} j_{n}-2 j_{n} J_{n}\right) \\
= & 2\left(D_{n}^{j} J_{n}+D_{n}^{J} j_{n}-2 J_{2 n}\right) .
\end{aligned}
$$

(2.29):

$$
\begin{aligned}
D_{n}^{j} D_{n}^{J}+\overline{D_{n}^{j}} \overline{D_{n}^{J}}= & \left(j_{n}+i j_{n+1}+j j_{n+2}+k j_{n+3}\right) \\
& \left(J_{n}+i J_{n+1}+j J_{n+2}+k J_{n+3}\right) \\
& +\left(j_{n}-i j_{n+1}-j j_{n+2}-k j_{n+3}\right) \\
& \left(J_{n}-i J_{n+1}-j J_{n+2}-k J_{n+3}\right) \\
= & 2 j_{n} J_{n}=2 J_{2 n} .
\end{aligned}
$$

In proofs, the identities of Jacobsthal and Jacobsthal-Lucas numbers given below were used, respectively,

$$
J_{m} j_{n}-J_{n} j_{m}=(-1)^{n} 2^{n+1} J_{m-n}, j_{n} J_{n}=J_{2 n} \text { and } j_{n+2}=j_{n+1}+2 j_{n} .
$$

Theorem 2.6. (Binet's Formula). Let $D_{n}^{J}$ and $D_{n}^{j}$ be $n$-th terms of dual Jacobsthal quaternion sequence $\left(D_{n}^{J}\right)$ and the dual Jacobsthal-Lucas quaternion sequence $\left(D_{n}^{j}\right)$, respectively. For $n \geq 1$, Binet's formula for these quaternions are as follows respectively,

$$
D_{n}^{J}=\frac{1}{\alpha-\beta}\left[\underline{\alpha} \alpha^{n}-\underline{\beta} \beta^{n}\right]
$$

and

$$
D_{n}^{j}=\left(\underline{\underline{\alpha}} \alpha^{n}+\underline{\underline{\beta}} \beta^{n}\right)
$$

where

$$
\begin{aligned}
\underline{\alpha} & =1+i(1-\beta)+j(3-\beta)+k(5-3 \beta), \alpha=2, \\
& \underline{\beta}=1+i(1-\alpha)+j(3-\alpha)+k(5-3 \alpha), \beta=-1, \\
\underline{\underline{\alpha}} & =(1-2 \beta)+i(5-\beta)+j(7-5 \beta)+k(17-7 \beta), \alpha=2, \\
\underline{\beta} & =(2 \alpha-1)+i(\alpha-5)+j(5 \alpha-7)+k(7 \alpha-17), \beta=-1 .
\end{aligned}
$$

Proof. The characteristic equation of recurrence relations $D_{n+2}^{J}=D_{n+1}^{J}+2 D_{n}^{J}$ is

$$
t^{2}-t-2=0 .
$$

The roots of this equation are $\alpha=2$ and $\beta=-1$

where $\alpha+\beta=1, \alpha-\beta=3, \alpha \beta=-2$.

Using recurrence relation and initial values $D_{0}^{J}=(0,1,1,3)$,

$D_{1}^{J}=(1,1,3,5)$ the Binet's formula for $D_{n}^{J}$, we get

$$
D_{n}^{J}=A \alpha^{n}+B \beta^{n}=\frac{1}{3}\left[\underline{\alpha} \alpha^{n}-\underline{\beta} \beta^{n}\right],
$$


where $A=\frac{D_{1}^{J}-D_{0}^{J} \beta}{\alpha-\beta}, B=\frac{\alpha D_{0}^{J}-D_{1}^{J}}{\alpha-\beta}$ and

$\underline{\alpha}=1+i(1-\beta)+j(3-\beta)+k(5-3 \beta), \underline{\beta}=1+i(1-\alpha)+j(3-\alpha)+k(5-3 \alpha)$. Similarly, using recurrence relations $D_{n+2}^{j}=D_{n+1}^{j}+2 D_{n}^{j}$, the Binet's formula for $D_{n}^{j}$ is obtained as follows:

$$
D_{n}^{j}=\left(\underline{\underline{\alpha}} \alpha^{n}+\underline{\underline{\beta}} \beta^{n}\right)
$$

Theorem 2.7. (Honsberger Identity) For $n, m \geq 0$ the Honsberger identity for the dual Jacobsthal quaternions $D_{n}^{J}$ and $D_{m}^{J}$ is given by

$$
D_{n}^{J} D_{m}^{J}+2 D_{n-1}^{J} D_{m-1}^{J}=2 D_{n+m-1}^{J}-J_{n+m-1} .
$$

Proof. (2.32):

$$
\begin{aligned}
D_{n}^{J} D_{m}^{J}= & J_{n} J_{m}+i\left(J_{n} J_{m+1}+J_{n+1} J_{m}\right)+j\left(J_{n} J_{m+2}+J_{n+2} J_{m}\right) \\
& +k\left(J_{n} J_{m+3}+J_{n+3} J_{m}\right)
\end{aligned}
$$

and

$$
\begin{aligned}
2 D_{n-1}^{J} D_{m-1}^{J}= & 2\left(J_{n-1} J_{m-1}\right)+2 i\left(J_{n-1} J_{m}+J_{n} J_{m-1}\right) \\
& +2 j\left(J_{n-1} J_{m+1}+J_{n+1} J_{m-1}\right) \\
& +2 k\left(J_{n-1} J_{m+2}+J_{n+2} J_{m-1}\right)
\end{aligned}
$$

Finally, adding equations (2.33) and (2.34) side by side, we obtain

$$
\begin{aligned}
D_{n}^{J} D_{m}^{J}+2 D_{n-1}^{J} D_{m-1}^{J}= & J_{n+m-1}+i\left(2 J_{n+m}\right) \\
& +j\left(2 J_{n+m+1}\right)+k\left(2 J_{n+m+2}\right) \\
= & 2 D_{n+m-1}^{J}-J_{n+m-1} .
\end{aligned}
$$

where the identity $J_{n+m}=J_{m} J_{n+1}+2 J_{m-1} J_{n}$ was used [27] and [28].

Theorem 2.8. D'ocagne's Identity For $n, m \geq 0$ the D'ocagne's identity for the dual-complex Jacobsthal quaternions $D_{n}^{J}$ and $D_{m}^{J}$ is given by

$$
D_{m}^{J} D_{n+1}^{J}-D_{m+1}^{J} D_{n}^{J}=(-1)^{n} 2^{n} J_{m-n}(1+i+5 j+7 k) .
$$

Proof. (2.35):

$$
\begin{aligned}
D_{m}^{J} D_{n+1}^{J}-D_{m+1}^{J} D_{n}^{J}= & {\left[\left(J_{m} J_{n+1}-J_{m+1} J_{n}\right)\right] } \\
& +i\left[\left(J_{m} J_{n+2}-J_{m+1} J_{n+1}\right)+\left(J_{m+1} J_{n+1}-J_{m+2} J_{n}\right)\right] \\
& +j\left[\left(J_{m} J_{n+3}-J_{m+1} J_{n+2}\right)+\left(J_{m+2} J_{n+1}-J_{m+3} J_{n}\right)\right] \\
& +k\left[\left(J_{m} J_{n+4}-J_{m+1} J_{n+3}\right)+\left(J_{m+3} J_{n+1}-J_{m+4} J_{n}\right)\right] \\
= & (-1)^{n} 2^{n} J_{m-n}(1+i+5 j+7 k) .
\end{aligned}
$$

where the identity $J_{m} J_{n+1}-J_{m+1} J_{n}=(-1)^{n} 2^{n} J_{m-n}$ was used [27] and [28].

Theorem 2.9. (Cassini's Identity). Let $D_{n}^{J}$ and $D_{n}^{j}$ be $n-$ th terms of dual Jacobsthal quaternion sequence $\left(D_{n}^{J}\right)$ and the dual Jacobsthal-Lucas quaternion sequence $\left(D_{n}^{j}\right)$, respectively. Then, we have

$$
\begin{aligned}
& D_{n-1}^{J} D_{n+1}^{J}-\left(D_{n}^{J}\right)^{2}=(-1)^{n} 2^{n-1}(1+i+5 j+7 k) . \\
& D_{n-1}^{j} D_{n+1}^{j}-\left(D_{n}^{j}\right)^{2}=(-2)^{n-1} 3^{2}(1+i+5 j+7 k) .
\end{aligned}
$$


Proof. (2.36):

$$
\begin{aligned}
D_{n-1}^{J} D_{n+1}^{J}-\left(D_{n}^{J}\right)^{2}= & \left(J_{n-1} J_{n+1}-J_{n}^{2}\right) \\
& +i\left(J_{n-1} J_{n+2}-J_{n} J_{n+1}\right) \\
& +j\left(J_{n-1} J_{n+3}-2 J_{n} J_{n+2}+J_{n+1}^{2}\right) \\
& +k\left(J_{n-1} J_{n+4}+J_{n+1} J_{n+2}-2 J_{n} J_{n+3}\right) \\
= & \left(J_{n-1} J_{n+1}-J_{n}^{2}\right) \\
& +i\left(J_{n-1} J_{n+1}-J_{n}^{2}\right) \\
& +5 j\left(J_{n-1} J_{n+1}-J_{n}^{2}\right) \\
& +7 k\left(J_{n-1} J_{n+1}-J_{n}^{2}\right) \\
= & (-1)^{n} 2^{n-1}(1+i+5 j+7 k) .
\end{aligned}
$$

and (2.37):

$$
\begin{aligned}
D_{n-1}^{j} D_{n+1}^{j}-\left(D_{n}^{j}\right)^{2}= & \left(j_{n-1} j_{n+1}-j_{n}^{2}\right) \\
& +i\left(j_{n-1} j_{n+2}-j_{n} j_{n+1}\right) \\
& +j\left(j_{n-1} j_{n+3}-2 j_{n} j_{n+2}+j_{n+1}^{2}\right) \\
& +k\left(j_{n-1} j_{n+4}+j_{n+1} j_{n+2}-2 j_{n} j_{n+3}\right) \\
= & \left(j_{n-1} j_{n+1}-j_{n}^{2}\right) \\
& +i\left(j_{n-1} j_{n+1}-j_{n}^{2}\right) \\
& +5 j\left(j_{n-1} j_{n+1}-j_{n}^{2}\right) \\
& +7 k\left(j_{n-1} j_{n+1}-j_{n}^{2}\right) \\
= & (-2)^{n-1} 3^{2}(1+i+5 j+7 k) .
\end{aligned}
$$

where identities of Jacobsthal numbers and Jacobsthal-Lucas numbers as follows:

$$
\begin{array}{cc}
J_{m} J_{n-1}-J_{m-1} J_{n}=(-1)^{n} 2^{n-1} J_{m-n}, & J_{n+2}=J_{n+1}+2 J_{n} \\
j_{m} j_{n-1}-j_{m-1} j_{n}=(-2)^{n-1} 3^{2} j_{m-n}, & j_{n+2}=j_{n+1}+2 j_{n} .
\end{array}
$$

were used [27] and [28].

Theorem 2.10. (Catalan's Identity). Let $D_{n}^{J}$ and $D_{n}^{j}$ be $n-$ th terms of dual Jacobsthal quaternion sequence $\left(D_{n}^{J}\right)$ and the dual Jacobsthal-Lucas quaternion sequence $\left(D_{n}^{j}\right)$, respectively. Then, we have

$$
\begin{aligned}
& D_{n+r}^{J} D_{n-r}^{J}-\left(D_{n}^{J}\right)^{2}=-(-2)^{n-r} J_{r}^{2}(1+i+5 j+7 k) . \\
& D_{n+r}^{j} D_{n-r}^{j}-\left(D_{n}^{j}\right)^{2}=-(-2)^{n-r} 3^{2} j_{r}^{2}(1+i+5 j+7 k) .
\end{aligned}
$$

Proof. (2.38):

$$
\begin{aligned}
D_{n+r}^{J} D_{n-r}^{J}-\left(D_{n}^{J}\right)^{2}= & \left(J_{n+r} J_{n-r}-J_{n}^{2}\right) \\
& +i\left[\left(J_{n+r} J_{n-r+1}-J_{n} J_{n+1}\right)\right. \\
& +\left(J_{n+r+1} J_{n-r}-J_{n+1} J_{n}\right) \\
& +j\left[\left(J_{n+r} J_{n-r+2}-J_{n} J_{n+2}\right)\right. \\
& \left.+\left(J_{n+r+2} J_{n-r}-J_{n+2} J_{n}\right)\right] \\
& +k\left[\left(J_{n+r} J_{n-r+3}-J_{n} J_{n+3}\right)\right. \\
& \left.+\left(J_{n+r+3} J_{n-r}-J_{n+3} J_{n}\right)\right] \\
= & -(-2)^{n-r} J_{r}^{2}(1+i+5 j+7 k) .
\end{aligned}
$$

and (2.39):

$$
\begin{aligned}
D_{n+r}^{j} D_{n-r}^{j}-\left(D_{n}^{j}\right)^{2}= & \left(j_{n+r} j_{n-r}-j_{n}^{2}\right) \\
& +i\left[\left(j_{n+r} j_{n-r+1}-j_{n} j_{n+1}\right)+\left(j_{n+r+1} j_{n-r}-j_{n+1} j_{n}\right)\right] \\
& +j\left[\left(j_{n+r} j_{n-r+2}-j_{n} j_{n+2}\right)+\left(j_{n+r+2} j_{n-r}-j_{n+2} j_{n}\right)\right] \\
& +k\left[\left(j_{n+r} j_{n-r+3}-j_{n+j_{n+3}}\right)+\left(j_{n+r+3} j_{n-r}-j_{n+3} j_{n}\right)\right] \\
= & -(-2)^{n-r} 3^{2} J_{r}^{2}(1+i+5 j+7 k) .
\end{aligned}
$$


where identities of Jacobsthal numbers and Jacobsthal-Lucas numbers as follows:

$$
\begin{gathered}
J_{n+r} J_{n-r}-J_{n} J_{n}=-(-2)^{n-r} J_{r}^{2} \\
j_{n+r} j_{n-r}-j_{n} j_{n}=(-2)^{n-r} 3^{2} J_{r}^{2} .
\end{gathered}
$$

were used [29].

\section{Conclusion}

The difference between the Jacobsthal and the dual Jacobsthal quaternions originated from the quaternionic units, i.e., the quaternionic units for the Jacobsthal quaternion are

$$
i^{2}=j^{2}=k^{2}=-1, i j=-j i=k, j k=-k j=i, \quad k i=-i k=j
$$

whereas for the dual Jacobsthal quaternions they are

$$
i^{2}=j^{2}=k^{2}=i j k=0, i j=-j i=j k=-k j=k i=-i k=0 .
$$

The set $J_{D}$ forms a commutative ring under the dual Jacobsthal quaternion multiplication and also it is a vector space of dimensions four on $\mathrm{R}$ and its basis is the set $\{1, i, j, k\}$. The interesting property of dual Jacobsthal quaternions is that by their means one can express the Galilean transformation in one quaternion equation. Since the multiplication and ratio of two dual Jacobsthal quaternions $D_{n}^{J_{1}}$ and $D_{n}^{J_{2}}$ is again a dual Jacobsthal quaternion, the set of dual Jacobsthal quaternions form a division algebra under addition and multiplication. There have been several studies on curve theory and magnetism by using the isomorphism between dual quaternion space and Galilean space $G^{4}$. Similar applications for dual Jacobsthal and dual Jacobsthal-Lucas quaternions can be applied to these areas.

Galilean transformation expressed by the dual four-component numbers shows the linkage between the space and time exists in the Newtonian physics. Moreover, it may have a considerable heuristic value for the study of the underlying mathematical formalism of physical laws. This study fills the gap in the literature by providing dual Jacobsthal quaternions.

\section{References}

[1] W. R. Hamilton, Elements of Quaternions, Longmans, Green and Co., London, (1866).

[2] A. F. Horadam, Complex Fibonacci numbers and Fibonacci quaternions. American Math. Monthly. 70(3)(1963), 289-291.

[3] M. R. Iyer, A note on Fibonacci quaternions. Fibonacci Quart., 7(3) (1969), 225-229.

[4] M. R. Iyer, Some results on Fibonacci quaternions, Fibonacci Quart.. 7 (1969), 201-210.

[5] E. Verner, Jr. Hoggatt, Fibonacci and Lucas Numbers, The Fibonacci Association, (1969).

[6] M. N. Swamy, On generalized Fibonacci quaternions, Fibonacci Quart., 11(5) (1973), 547-550.

[7] A. L. Iakin, Generalized Quaternions of higher order, Fibonacci Quart., 15(4) (1977), 343-346.

[8] A. L. Iakin, Generalized Quaternions with quaternion components, Fibonacci Quart., 15 (1977), 350-352.

[9] C. J. Harman, Complex Fibonacci numbers, Fibonacci Quart., 19(1) (1981), 82-86.

[10] A. F. Horadam, Quaternion recurrence relations, Ulam Quarterly. 2(2) (1993), 23-33.

[11] A. F. Horadam, A generalized Fibonacci sequence, Amer. Math. Month., 68(5) (1961), 455-459.

${ }^{[12]}$ L. Kula, Y. Yayl1, Split Quaternions and rotations in semi-Euclidean space, J. Korean Math. Soc. 44(6) (2007), 1313-1327.

${ }^{[13]}$ E. Ata, Y. Yayl1, Dual quaternions and dual projective spaces, Chaos Solitons Fractals, 40(3) (2009), 1255-1263.

[14] S. Halıc1, On Fibonacci quaternions, Adv. Appl. Clifford Algebr., 22(2) (2012), 321-327.

[15] S. Halıc1, On Complex Fibonacci Quaternions, Adv. Appl. Clifford Algebr., 23 (2013), 105-112.

[16] M. Akyiğit, H. H. Kösal and M. Tosun, Split Fibonacci quaternions, Adv. Appl. Clifford Algebr., 23(3) (2013), 535-545.

[17] K. S. Nurkan, and A. İ. Güven, Dual Fibonacci quaternions, Adv. Appl. Clifford Algebr., (2014) doi: 10.1007/s00006-0140488-7

[18] V. Majernik, Quaternion formulation of the Galilean space-time transformation, Acta Phy. Slovaca., 56(1) (2006), 9-14. 
[19] V. Majernik, Galilean transformation expressed by the dual four-component numbers, Acta Phy. Polonica A., 87(6) (1995), 919-923.

[20] Z. Ercan, S. Yüce, On properties of the dual quaternions, Eur. J. Pure Appl. Math., 4(2) (2011), $142-146$.

[21] B. Artmann, The concept of Number: From Quaternions to Modals and topological Fields, Ellis Horwood, Chicherster, (1988).

[22] S. Yüce, F. Torunbalcı Aydın, A new aspect of dual Fibonacci quaternions, Adv. Appl. Clifford Algebr., 26(2) (2016), 873-884.

[23] N. J. A. Sloane, A Handbook of Integer Sequences, New York, Press, (1973).

[24] A. F. Horadam, Jacobsthal and Pell Curves, Fibonacci Quart., 26 (1988), 79-83.

[25] A. F. Horadam, Jacobsthal Representation Numbers, Fibonacci Quart., 34 (1996), 40-54.

[26] A. F. Horadam, Jacobsthal representation polynomials, Fibonacci Quart., 35 (1997), 137-148.

[27] F. Köken, D. Bozkurt, On the Jacobsthal numbers by matrix methods, Int. J. Contemp. Math. Sci., 3 (13), 605-614 (2008)

[28] F. Köken, D. Bozkurt, On the Jacobsthal-Lucas numbers by matrix methods, Int. J. Contemp. Math. Sci., 3(13) (2008), 1629-1633.

[29] A. Daşdemir, On the Jacobsthal numbers by matrix method, SDU J. Sci., 71 (2012), 69-76.

[30] G. B. Djordjevid, Generalized Jacobsthal polynomials, Fibonacci Quart., 38 (2009), 239-243.

[31] Z. Cerin, Sums of squares and products of Jacobsthal numbers, J. Integer Seq., 10 (2007), Article 07.2.5,.

[32] Z. Cerin, Formulae for sums of Jacobsthal-Lucas numbers, Int. Math. Forum., 2(40) (2007), 1969-1984.

[33] A. Szynal-Liana, I. Włoch, A note on Jacobsthal quaternions, Adv. Appl. Clifford Algebr., 26(1) (2016), $441-447$.

[34] F. Torunbalcı Aydın, S. Yüce, A new approach to Jacobsthal quaternions, Filomat, 31(18) (2017), 5567-5579.

[35] D. Taşç1, On k-Jacobsthal and k-Jacobsthal-Lucas quaternions, J. Sci. Arts, 3 (2017), 469-476.

[36] G. Cerda-Morales, Identities for third order Jacobsthal quaternions, Adv. Appl. Clifford Algebr., 27(2) (2017), 1043-1053.

[37] G. Cerda-Morales, On k-Jacobsthal and k-Jacobsthal-Lucas quaternions, J. Math. Sci. Model., 1(2) (2018), $73-79$. 